\title{
Fatores que influenciam os custos, na abordagem da gestão estratégica de custos: análise empírica em centros de saúde municipais*
}

Factores que influencian los costes, en el enfoque de la gestión estratégica de coste: análisis empírico en centros de salud municipales

Factors that influence costs, in the approach of strategic cost management: empirical analysis in municipal health

\author{
centers
}

Eduardo Augusto Borgert a

Universidade Federal de Santa Catarina, Brasil

eduardo@borgert.com.br

ORCID: http://orcid.org/0000-0002-5779-2796

Valdirene Gasparetto

Universidade Federal de Santa Catarina, Brasil

ORCID: http://orcid.org/0000-0002-2825-4067

DOI: https://doi.org/10.11144/Javeriana.cc20-49.fica

\section{Resumo:}

O presente estudo procura identificar fatores que influenciam os custos nos centros de saúde administrados pela Secretaria Municipal de Saúde de Florianópolis, SC, Brasil, segundo a Gestão Estratégica de Custos -GEC-. Uma revisão da literatura da GEC para o contexto pesquisado permitiu identificar as variáveis representativas de cada cost driver. Foi realizada uma pesquisa de levantamento nos 49 centros de saúde da SMS para relacionar as variáveis com o custo. Constatou-se, a partir das variáveis do cost driver "escala", que os centros de saúde que possuem maior demanda de pacientes necessitam de estruturas maiores, tendo um impacto mais rápido nos custos. Já outros cost drivers como "experiência" ou "Eficiência das Instalações" mostram uma incidência menor nos custos.

Codigos JEL: I19, M19, M41

Palavras-chave: Cost drivers, gestão estratégica de custos, centros de saúde municipais.

\section{Resumen:}

El presente estudio procura identificar factores que influencian los costos en los centros de salud administrados por la Secretaría Municipal de Salud de Florianópolis, SC, Brasil, según la Gestión Estratégica de costos -GEC-. Una revisión de la literatura sobre la GEC para el contexto investigado permitió identificar las variables representativas de cada cost driver. Fue realizado un levantamiento en los 49 centros de salud de la SMS para relacionar las variables con el costo. Se constató, a partir de las variables del cost driver "escala", que los centros de salud que poseen mayor demanda de pacientes necesitan de estructuras mayores, teniendo un impacto más rápido en los costos. Por otro lado, los cost drivers como "experiencia" o "eficiencia de las instalaciones" muestran una incidencia menor en los costos.

Códigos JEL: I19, M19, M41

Palabras clave: Cost drivers, gestión estratégica de costos, centros de salud municipales.

\section{Abstract:}

The purpose of this paper is to identify factors that influence the costs of the health centers administered by the Municipal Health Department of Florianópolis, SC, Brazil, using the approach of Strategic Cost Management -SCM-. Literature review and support from health experts allowed us to identify the variables that represent each cost driver, under the SCM approach. We carried out a survey in the 49 health centers to relate the variables to the cost. The results show that health centers with greater demand for patients need larger structures. In these situations, oscillations in the capacity have a faster impact on costs, while Experience and Plant Layout Efficiency act on a smaller portion of the cost and its impacts are noticed more slowly.

JEL Codes: I19, M19, M41

Keywords: Cost drivers, Strategic cost management, Health centers.

\section{Autor notes:}




\section{INTRODUÇÃO}

Nos anos recentes, pesquisas passaram a demonstrar que outras variáveis, além do volume de produção, podem direcionar ou determinar os custos das organizações. Essas novas variáveis vêm ganhando importância estratégica para os gestores no desenho dos sistemas de contabilidade gerencial. Dentre essas pesquisas, em 1993, Shank e Govindarajan, pesquisadores de contabilidade gerencial, verificando a necessidade de incorporar aspectos estratégicos na gestão dos custos, propuseram a Strategic Cost Management-SCM- ou Gestão Estratégica de Custos -GEC-. Esta abordagem está composta por três pilares: (i) análise da cadeia de valor, (ii) análise do posicionamento estratégico, e (iii) análise dos cost drivers (ou direcionadores de custos), classificados segundo Riley (1987) em estruturais e de execução. A análise de cost drivers na GEC tem o objetivo de identificar os fatores que influenciam nos custos de uma organização e utilizá-los no planejamento estratégico (Shank \& Govindarajan, 1993). Esses cost drivers, e suas relações com o valor ao consumidor (custos, receitas e lucratividade), são estrategicamente importantes para as empresas por conta de serem os principais alicerces das decisões estratégicas dos gestores (Porter, 1985).

Em reflexão sobre a GEC, Niu, Chen, Jiang e Zhou (2012) mencionam que seus objetivos devem estar, de um lado ligados aos objetivos estratégicos, e de outro alinhados às características do sistema de custos, em sua parte operacional. Neste último quesito o objetivo é uma redução de custos sustentável.

Liu (2015) menciona que a característica mais importante da GEC é a combinação entre gerenciamento dos custos e a estratégia. Quando as organizações estabelecem a estratégia competitiva, a estratégia de custos deve seguir e estar alinhada a esta.

Em áreas que envolvem o poder público, como a saúde, o melhor conhecimento dos fatores que afetam os custos permite que os recursos sejam utilizados da melhor maneira para atender as demandas da população. Hsu e Qu (2012) lembram que em alguns setores da economia, como na saúde, manter um sistema de custos alinhado auxilia no planejamento da organização em relação ao reembolso de suas atividades.

Em estudo com 120 hospitais na Bélgica, Cardinaels, Roodhooft e Herck (2004) constataram que os gastos com saúde estão em crescimento, enquanto os recursos para financiá-la são cada vez menores. No Brasil, Bonacim e Araújo (2010) observaram, na mesma linha, que há insuficiência desses recursos no setor público. Assim, os recursos necessários para a prestação da assistência médica são cada vez maiores em função das novas opções que a tecnologia oferece. A gestão dos custos nas organizações de saúde pode fornecer auxílio para utilizar de forma adequada esses recursos e atender à demanda.

A análise de cost drivers foi abordada em muitos contextos. Foster e Gupta (1990) realizaram um estudo em 37 indústrias do setor eletrônico americano para verificar se a complexidade e a eficiência afetavam o nível de custos indiretos das empresas. Igualmente, Banker e Johnston (1993) analisaram companhias aéreas norte-americanas e verificaram que o volume e a complexidade das operações são cost drivers representativos para este setor. Alcouffe Berland, Dreveton e Essid (2010) pesquisaram os cost drivers ambientais no ramo da engenharia civil e atribuíram pesos para cada fator encontrado por meio de escala Likert. Observa-se, porém, que há poucos estudos que analisam os fatores que direcionam os custos noutros contextos, como na área da saúde.

Neste sentido, este estudo tem como foco as Unidades Básicas de Saúde ou centros de saúde. Foram analisadas as unidades do Programa Saúde da Família -PSF- que prestam serviços de atenção básica à população nos municípios brasileiros, a partir dos princípios do Sistema Único de Saúde (Ministério da Saúde, 2008). Avaliando especificamente dados dos centros de saúde de Florianópolis, SC, este estudo responde à pergunta: qual a influência dos cost drivers da GEC nos custos de centros de saúde municipais?

Neste contexto, o presente estudo tem o objetivo de identificar os fatores que influenciam os custos, na abordagem da GEC, nos centros de saúde administrados pela Secretaria Municipal de Saúde de Florianópolis, SC. O estudo pretende estabelecer relações entre os cost drivers estruturais e de execução para o cenário estudado, oferecendo nova evidência empírica para em um contexto específico. 
Apesar da magnitude dos gastos em saúde, as organizações que prestam esses serviços ainda não têm muito conhecimento e controle sobre tais montantes. Segundo o Instituto Brasileiro de Geografia e Pesquisa (2017), em 2015, no Brasil esses gastos foram de R \$ 546 bilhões (9,1\% do PIB). De acordo com Alemi e Sullivan (2007), os gestores normalmente não conhecem os custos dos serviços prestados, o que pode prejudicar a gestão de custos e a própria competitividade do setor como um todo.

A análise de cost drivers é uma abordagem que pode contribuir para a definição de estratégias para o setor de saúde. Dieng, Araujo, Diniz, Diniz e Santos (2006) afirmam que conhecer os fatores que direcionam os custos oferece aos gestores oportunidades de gerenciar, melhorar e, consequentemente, reduzir custos. Isto se torna relevante num cenário onde a demanda de gastos e as necessidades da população estão em constante crescimento.

Anderson (2007) afirma que os estudos nessa linha não analisam os cost drivers, estruturais e de execução, de maneira conjunta, mas de forma individual. Além disso, Henri, Boiral e Roy (2015) mencionam que poucos estudos analisam questões relacionadas à gestão dos custos estruturais. Neste estudo são analisados de maneira conjunta os cost drivers estruturais e de execução conforme propostos por Riley (1987), com o fim de contribuir para o preenchimento da lacuna de pesquisa identificada.

\section{FUNDAMENTAÇÃO TEÓRICA}

\section{Cost drivers}

O termo cost driver ganhou relevância no final do Século XX, quando surgiram novas ferramentas de gestão e de custos em resposta à crescente demanda das organizações e da academia pela melhoria da qualidade das informações geradas pela contabilidade. Este fenômeno deu-se especialmente na contabilidade gerencial (Johnson \& Kaplan, 1987; Johnson, 1992). Essa preocupação ocasionou uma mudança de paradigma, que ganhou força a partir da década de 1980. Os autores alertavam que as práticas tradicionais de contabilidade gerencial não atendiam adequadamente as necessidades das organizações. Estas haviam perdido a capacidade de gerar informações relevantes à tomada de decisão em função das mudanças que ao longo de todo o século XX estavam ocorrendo na economia, na tecnologia e nas organizações (IFAC, 1998).

Neste cenário, o foco da contabilidade gerencial ampliou-se para uma perspectiva estratégica, possibilitando aos gestores melhor avaliar o atendimento aos clientes e o posicionamento das organizações na cadeia de valor. Nesse contexto, a área de custos também devia prover informações para auxiliar as empresas a alcançar e manter vantagem competitiva sustentável (Hansen \& Mowen, 2005). Autores como Kaplan (1983, 1984), Miller e Vollmann (1985), Cooper e Kaplan (1987), buscavam melhores formas de atribuir os custos indiretos aos produtos e aprimorar a gestão das organizações, e não apenas valorar os estoques para relatórios financeiros. Isto incentivou a preocupação com os cost drivers para alocação de custos aos objetos de custos; o denominado contexto operacional. Um dos primeiros estudos empíricos mencionando cost drivers com enfoque operacional foi de Miller e Vollmann (1985). Eles identificaram os cost drivers envolvidos nas empresas de cinco segmentos da indústria eletrônica, verificando que a verdadeira força que determina os custos indiretos não é o volume de produção, mas as transações envolvendo logística, equilíbrio (balanceamento entre produção e demanda), qualidade e mudanças no processo produtivo.

Noutra perspectiva, Porter (1985), Shank (1989) e Shank e Govindarajan (1989) ampliaram o escopo dos cost drivers utilizando uma abordagem mais estratégica para análise de custos, o que se denomina nesta pesquisa como cost drivers estratégicos. $\mathrm{O}$ termo cost driver tem sido utilizado para representar pelo menos três papeis na contabilidade gerencial: (i) determinar, em termos estratégicos, a estrutura de custos de uma organização (no contexto estratégico - da GEC); (ii) possibilitar a atribuição dos custos aos objetos de custos (no contexto operacional); e (iii) explicar o comportamento dos custos (cost behavior), no sentido de analisar 
como o custo total do objeto de custo se modifica conforme os fatores se alteram (Blocher, Chen $\&$ Lin, 2008; Horngren, Datar \& Foster, 1999; Morse, Davis \& Hartgraves, 2002). O que distingue os significados do termo cost driver em suas diferentes abordagens são o campo de conhecimento, o propósito a que se destina e o foco a ser analisado em cada estudo.

Do ponto de vista da gestão estratégica de custos, o termo cost driver representa um dos elementos no processo de planejamento estratégico. Igualmente, pode ter um papel central na definição do posicionamento das organizações em seu meio, ao servir de fonte de vantagem competitiva (Porter, 1985). Esta abordagem é aparentemente menos disseminada do que aquelas associadas à atribuição dos custos (contexto operacional) e o estudo de seu comportamento.

$\mathrm{Na}$ tentativa de definir as diferentes abordagens acerca do termo cost driver, Banker e Johnston (2007) trazem as principais vertentes utilizadas nas pesquisas no âmbito da contabilidade gerencial (tabela 1).

TABLA 1

Comparação das diferentes taxonomias dos cost drivers

\begin{tabular}{|c|c|c|}
\hline Porter (1985) & Riley (1987) & Cooper e Kaplan (1998) \\
\hline Economias de & Estruturais & Fase de fabricação da \\
\hline escala & Escala & cadeia de valor \\
\hline Aprendizagem & Escopo & Nível de unidade \\
\hline Utilização da & Tecnologia & Nível do lote \\
\hline capacidade & Experiência & Suporte do produto \\
\hline Elos & Complexidade & Suporte das instalações \\
\hline Inter-relações & Execução & Restante da cadeia de \\
\hline Integração & Envolvimento & valor \\
\hline Momento & força de trabalho & Suporte ao cliente \\
\hline oportuno & Gestão da qualidade & Suporte da linha \\
\hline Políticas & Utilização da & produtos \\
\hline discricionárias & capacidade & Suporte da marca \\
\hline Localização & Eficiência do layout & Suporte de canal \\
\hline Fatores & das instalações & Suporte de localização \\
\hline institucionais & Configuração & Suporte corporativo \\
\hline & produto & Cadeia de valor ampliada \\
\hline & Exploração & Suporte do formecedor \\
\hline & ligações & \\
\hline
\end{tabular}

Fonte: adaptado de Banker e Johnston (2007).

Conforme a tabela 1, os principais influenciadores para as pesquisas com abordagem estratégica de cost drivers são Porter (1985) e Riley (1987), ambos da área de planejamento estratégico. Porter (1985), baseado em teorias econômicas industriais, definiu cost drivers como sendo os "determinantes estruturais dos custos das atividades organizacionais". Riley (1987) aprimorou a definição de Porter (1985), propondo duas categorias distintas para os cost drivers: estruturais e de execução. Essa abordagem é utilizada como um dos pilares do que posteriormente definiu-se como a gestão estratégica de custos, o principal instrumento das pesquisas em cost drivers estratégicos.

A abordagem de Cooper e Kaplan $(1987,1998)$ desenvolveu os modelos Activity-Based Costing (ABC) e no Activity-Based Management (ABM). Ambos instrumentos têm a finalidade de melhorar a alocação de custos aos objetos de custos a partir dos direcionadores de atividades. No trabalho de 1998, eles atribuíram a nomenclatura de process drivers para denominar esses direcionadores de atividades - os cost drivers operacionais (Banker \& Johnson, 2007). 
A discussão a respeito da construção teórica dos cost drivers é estendida para a literatura brasileira. Nela se observa o uso do termo cost drivers como conceito estratégico, mas muitas vezes abordando os direcionadores operacionais. Assim, a taxonomia, em português, para identificar os cost drivers estratégicos, seguindo a Porter (1985), Riley (1987) e Shank e Govindarajan (1993), seriam "determinantes de custos". Já os process drivers ou cost drivers operacionais seriam definidos como "direcionadores de custos" (Slavov \& Takahashi, 2010).

\section{Cost drivers estratégicos}

Porter (1985) definiu dez categorias principais de cost drivers estratégicos. Riley (1987) complementou o modelo, propondo novos cost drivers estratégicos, separados em duas categorias: estruturais e de execução. Posteriormente, Shank e Govindarajan (1993) incorporam esses conceitos de Riley (1987) como um dos pilares do seu esquema da GEC. Na perspectiva dos cost drivers estruturais, as escolhas estratégicas estão relacionadas à estrutura econômica da empresa. Os cost drivers de execução, entretanto, são os determinantes da posição de custos de uma empresa que dependem de sua capacidade de executar de forma bem-sucedida.

Para Shank e Govindarajan (1997), os cost drivers estruturais não são diretamente relacionados ao desempenho, pois nem sempre mais significa melhor. Uma linha de produtos complexa não é necessariamente melhor do que uma menos complexa. Já para os cost drivers de execução, mais sempre significa melhor.

Costa e Rocha (2014), em levantamento dos principais cost drivers estratégicos, identificaram sua influência nas empresas produtoras do setor de eletroeletrônicos no Brasil, utilizando exclusivamente informações públicas. Verificaram que os cost drivers podem ser de cunho mais qualitativo do que quantitativo. Isto é, as informações indicam a existência de um fator determinante de custo, mas que nem sempre é suficiente para explicar o que causa o custo. Igualmente, eles destacam que grande parte dos trabalhos que analisam cost drivers utiliza a abordagem de Shank e Govindarajan (1993) da GEC. No entanto, não há consenso nesses estudos, e a lista poderia se estender quase infinitamente, dada a quantidade de fatores que podem ter influência sobre os custos.

Estudos posteriores analisaram conjuntos de cost drivers que nem sempre remetem à classificação proposta por Riley (1987), mas consideram aspectos semelhantes. Foster e Gupta (1990), além da estrutura e execução, consideram o volume, a complexidade e a eficiência, em 37 indústrias do setor eletrônico dos Estados Unidos. Eles verificaram que estes fatores impactam no nível de overheads destas organizações, mas não puderam capturar todas as variáveis que determinam os custos. Os resultados sugerem uma alta correlação entre os custos indiretos e o volume de produção, e uma baixa correlação quando se considera eficiência e complexidade.

Banker e Johnston (1993) verificaram que o volume e complexidade das operações são cost drivers representativos para o setor das companhias aéreas norte-americanas. Datar, Kekre, Mukhopadhyay \& Srinivasan (1993) analisaram os cost drivers associados aos custos indiretos na indústria de lâmpadas de automóveis. Para isto, utilizaram equações estruturais que estimam o relacionamento entre variáveis que medem o volume de produção e a complexidade. As variáveis envolvendo complexidade foram as mais significativas para o custo.

Banker, Potter e Schroeder (1995) analisaram o impacto do volume nos custos indiretos de empresas do ramo eletrônico dos EUA. Encontrou-se uma relação forte e positiva entre custo e volume, porém, constataram que a maior parte da variação nos custos indiretos pode ser explicada por operações de fabricação, e não necessariamente pelo volume. Leitch (2001) verificou que o escopo, a capacidade e o prazo de entrega são determinantes que devem ser geridos na gestão de custos. Horngren, Bhimani, Datar e Foster (2002) verificaram que as variáveis do nível de atividade ou volume afetam os custos ao longo de um período de tempo.

Alcouffe et al. (2010) desenvolveram um framework para os cost drivers ambientais no ramo da engenharia civil. Utilizaram a literatura existente de cost drivers, assim como a literatura na área ambiental, para definir o conjunto de variáveis. Concluíram que os cost drivers da tecnologia e da complexidade afetam fortemente a 
posição de custos das empresas pesquisadas. Também analisando o cost driver da tecnologia, Diehl, Miotto e Souza (2010) verificaram a sua influência direta no setor aéreo brasileiro, no período de 1997 a 2005.

Apesar das diferentes abordagens sobre cost drivers, as publicações nesta área não parecem seguir uma linha definida. Portanto determina-se, para o escopo deste trabalho, o agrupamento da GEC na abordagem de Riley (1987). Desta forma são abordados apenas os cost drivers pertencentes a este framework, subdivididos em estruturais e de execução.

\section{PROCEDIMENTOS METODOLÓGICOS}

\section{População e amostra}

Este estudo compreende os centros de saúde de Florianópolis, Estado de Santa Catarina, no Brasil. Atualmente, a Secretaria Municipal de Saúde -SMS- de Florianópolis administra 49 unidades de saúde, que atendem a população do município com recursos do Sistema Único de Saúde. Os centros de saúde administrados pela SMS de Florianópolis estão inseridos na estratégia do Programa de Saúde da Família PSF-. Este programa é operado pelas Equipes de Saúde da Família, responsáveis, cada uma, por cuidar cerca de 4 mil habitantes de sua região. Cada centro de saúde é instalado nos bairros considerando o tamanho da população.

No contexto da estratégia do PSF, a SMS de Florianópolis é responsável por administrar os centros de saúde, a partir da Diretoria de Atenção à Saúde, segregada em três subdivisões: (i) gerência de atenção primária, (ii) gerência de atenção especializada, e (iii) gerência de integração assistencial.

A gerência de atenção primária é responsável pelas unidades básicas de saúde, onde estão inseridos os centros de saúde. Dentro da lógica da estratégia do PSF, os centros de saúde servem como "porta de entrada" para os pacientes. Estes são avaliados conforme necessidade, podendo ser tratados nos próprios centros de saúde ou encaminhados para a atenção especializada. A gerência de atenção especializada é a responsável pelas Policlínicas, Centros de Especialidade Odontológica, Unidades de Pronto Atendimento e Serviço de Atendimento Móvel de Urgência. Por fim, a gerência de integração assistencial é responsável pelo Centro de Atenção Psicossocial, Núcleo de Apoio à Saúde da Família e pela Farmácia Escola.

A amostra da pesquisa compreende os 49 centros de saúde e a pesquisa utiliza dados referentes ao ano de 2016. Os relatórios do ano de 2017 ainda não haviam sido disponibilizados até a data da coleta dos dados desta pesquisa.

\section{Procedimentos para coleta dos dados}

Foram identificadas as variáveis representativas de cada cost driver da abordagem de Riley (1987) e adaptadas ao contexto de saúde. Para cada variável identificou-se a forma pela qual poderia ser mensurada. Dois especialistas, atuantes na SMS de Florianópolis e envolvidos na administração dos centros de saúde, analisaram as variáveis e a forma de mensuração, e fizeram a validação, resultando nos dados constantes na tabela 2. 
TABLA 2

Cost drivers, variáveis e forma de mensuração para os centros de saúde da pesquisa

\begin{tabular}{|c|c|c|c|}
\hline $\mathrm{N}^{\circ}$ & Cost driver & $\begin{array}{l}\text { Variáveis no contexto dos } \\
\text { centros de saúde de } \\
\text { Florianópolis }\end{array}$ & $\begin{array}{l}\text { Forma de mensuração com dados } \\
\text { de cada centro de saúde }\end{array}$ \\
\hline 1 & \multirow{6}{*}{ Escala } & Número de funcionários & Número de funcionários \\
\hline 2 & & Tamanho da estrutura física & Area das instalações (em m2) \\
\hline 3 & & Investimento em estrutura & Investimento em estrutura (em R $\$$ ) \\
\hline 4 & & $\begin{array}{c}\text { Número de equipes de saúde } \\
\text { da familia }\end{array}$ & $\begin{array}{c}\text { Número de equipes de saúde da } \\
\text { família }\end{array}$ \\
\hline 5 & & $\begin{array}{l}\text { Investimento em insumose } \\
\text { medicamentos }\end{array}$ & $\begin{array}{l}\text { Gastos com insumose } \\
\text { medicamentos (em R } \$ \text { ) }\end{array}$ \\
\hline 6 & & Número de atendimentos & Número de atendimentos realizados \\
\hline 7 & Escopo & $\begin{array}{c}\text { Número de especialidades } \\
\text { atendidas }\end{array}$ & $\begin{array}{l}\text { Número de diferentes } \\
\text { especialidades atendidas }\end{array}$ \\
\hline 8 & \multirow{3}{*}{ Experiência } & Resolutividade de pacientes & $\begin{array}{l}\text { Nivel de resolutividade dos casos } \\
\text { (em \%) }\end{array}$ \\
\hline 9 & & Tempo médio de atendimento & $\begin{array}{l}\text { Total de horas de consultas / } \\
\text { número de pacientes atendidos }\end{array}$ \\
\hline 10 & & $\begin{array}{l}\text { Médicos com residência em } \\
\text { saúde da família }\end{array}$ & $\begin{array}{l}\text { Percentual dos médicos com } \\
\text { residência em saúde da família }\end{array}$ \\
\hline 11 & Tecnologia & Idade média das instalações & $\begin{array}{l}\text { Tempo desde a última reforma ejou } \\
\text { inauguração da unidade (em anos) }\end{array}$ \\
\hline 12 & $\begin{array}{l}\text { Comprometimento } \\
\text { da força de } \\
\text { trabalho }\end{array}$ & $\begin{array}{l}\text { Número médio de dias } \\
\text { trabalhados por funcionário }\end{array}$ & $\begin{array}{l}\text { Número médio de dias trabalhados } \\
\text { por funcionário }\end{array}$ \\
\hline 13 & \multirow{2}{*}{$\begin{array}{l}\text { Gestão da } \\
\text { qualidade total }\end{array}$} & Índice "hiperutilizadores" & $\begin{array}{l}\text { Utilizadores com mais de } 7 \\
\text { consultas em um mês (em \%) }\end{array}$ \\
\hline 14 & & $\begin{array}{c}\text { Número de reclamações na } \\
\text { ouvidoria }\end{array}$ & $\begin{array}{c}\text { Número de reclamações na } \\
\text { ouvidoria }\end{array}$ \\
\hline 15 & \multirow{3}{*}{$\begin{array}{l}\text { Utilização da } \\
\text { capacidade }\end{array}$} & Faltas em consultas & \multirow{3}{*}{$\begin{array}{c}\text { \% de faltas em consultas } \\
\text { Número total de consultas } / \mathrm{m}^{2} \text { das } \\
\text { instalações } \\
\text { \% de custos fixos em relação ao } \\
\text { custo total }\end{array}$} \\
\hline 16 & & $\begin{array}{l}\text { Número de atendimentos por } \\
\qquad \mathrm{m}^{2}\end{array}$ & \\
\hline 17 & & Percentual dos custos fixos & \\
\hline 18 & \multirow{2}{*}{$\begin{array}{l}\text { Eficiência do } \\
\text { layout das } \\
\text { instalações }\end{array}$} & Índice da população atendida & $\begin{array}{c}\text { Número de atendimentos a } \\
\text { diferentes pacientes / população da } \\
\text { área de abrangência }\end{array}$ \\
\hline 19 & & $\begin{array}{c}\text { Distância média do centro de } \\
\text { saúde para área de } \\
\text { abrangência }\end{array}$ & $\begin{array}{c}\text { Distância média das residências } \\
\text { para o centro de saúde (área em } \\
\mathrm{km}^{2} \text { ) }\end{array}$ \\
\hline 20 & $\begin{array}{l}\text { Configuração do } \\
\text { produto }\end{array}$ & $\begin{array}{c}\text { Nota no PMAQ (Programa } \\
\text { Nacional de Melhoria do } \\
\text { Acesso e da Qualidade da } \\
\text { Atenção Básica) }\end{array}$ & $\begin{array}{c}\text { Avaliação para cada unidade } \\
\text { (1=mediano ou abaixo da média; } \\
\text { 3=acima da média; e } 5=\text { muito } \\
\text { acima da média) }\end{array}$ \\
\hline
\end{tabular}

Fonte: elaborado pelos autores.

A validação com os especialistas levou à eliminação de algumas variáveis e inclusão de outras, devido às características das operações nos centros de saúde. Desta forma, não puderam ser medidos os cost drivers "Complexidade" e "Exploração das Ligações na Cadeia de Valor". As variáveis incluídas no modelo foram "Médicos com Residência em Saúde da Família” e "Nota no PMAQ”. Segundo os especialistas, os médicos que realizaram residência na área de saúde da família possuem melhor capacidade técnica para atuar na atenção básica e, portanto, se enquadram no cost driver "Experiência". Já, a "Nota no PMAQ" se enquadra no cost driver "Configuração do Produto", pois é um programa que avalia a atuação das equipes de saúde e oferece uma avaliação da prestação do serviço ao paciente.

Os custos dos 49 centros de saúde pesquisados correspondem aos custos diretos de cada centro, e foram obtidos a partir de informações disponibilizadas pela SMS. A coleta dos dados foi feita com análise 
documental dos centros de saúde. Os dados foram coletados a partir de relatórios fornecidos pela gerência de planejamento da SMS, sendo a maioria dados públicos.

\section{Procedimentos para análise dos dados}

A partir dos dados coletados conforme as variáveis explicitadas na tabela 2, elaborou-se a Análise Fatorial (AF), para agrupar as variáveis e melhor identificar suas relações. A Análise Fatorial é uma técnica estatística que busca, pela avaliação de um conjunto de variáveis, identificar as dimensões de variabilidade comuns existentes em um conjunto de fenômenos. A AF é utilizada com o intuito de desvendar estruturas existentes (os fatores), mas que não são observáveis diretamente (Bezerra, 2014).

A partir dos fatores, ou dimensões, resultados da análise fatorial são geradas novas variáveis no modelo. Com esta etapa se espera eliminar a multicolinearidade dos dados, para que seja possível realizar o próximo passo da análise. A segunda etapa da análise consistiu em relacionar os fatores gerados pela Análise Fatorial e confronta-os com os custos diretos dos centros de saúde do município de Florianópolis. Isto permite verificar sua associação, a partir da regressão linear múltipla, e observar como os cost drivers influenciam nos custos dos centros de saúde.

\section{APRESENTAÇÃO E ANÁLISE DOS RESULTADOS DA PESQUISA}

\section{Análise dos cost drivers por meio dos fatores}

Para verificar a adequação da amostra foi realizado teste de Kaiser-Meyer-Olklin (KMO), em que os valores variam entre 0 e 1 , bem como a estatística Bartlett Test of Spherecity (BTS), que deve ser estatisticamente significante $(\mathrm{p}<0,05)$, conforme tabela 3 .

TABLA 3

Teste de KMO e Bartlett

\begin{tabular}{c|l|l}
\hline Medida Kaiser-Meyer-Olkin (KMO) de adequação de amostragem & 0,663 \\
\hline \multirow{2}{*}{$\begin{array}{c}\text { Teste de esfericidade de } \\
\text { Bartlett }\end{array}$} & Gl & 307,270 \\
\cline { 2 - 3 } & Sig. & 91 \\
\hline
\end{tabular}

Fonte: dados da pesquisa.

$\mathrm{O}$ índice de $\mathrm{KMO}$, acima do índice crítico de 0,5 , indica que o resultado é satisfatório para utilizar a AF. O nível de significância no teste de Bartlett também ficou dentro do recomendado - menor que 0,05. As variáveis “\% de Faltas em Consultas", "Número de Especialidades Atendidas”, "Tempo Médio de Atendimento", "Número de Atendimentos por $\mathrm{m}^{2}$ ", "Distância Média do Centro de Saúde para Área de Abrangência" e "Idade Média das Instalações" foram excluídas por não apresentarem valores dentro dos intervalos desejados para a $\mathrm{AF}$, o que resultou em um modelo com um total de 14 variáveis.

$\mathrm{Na}$ sequência se verificou o total de variância que o modelo atual consegue explicar. A tabela 4 mostra os resultados desta análise. 
TABLA 4

Variância total explicada

\begin{tabular}{c|c|c|c}
\hline \multicolumn{4}{c}{ Autovalores iniciais } \\
\hline Componente & Total & \% de variância & \% cumulativa \\
\hline 1 & 5,029 & 35,920 & 35,920 \\
\hline 2 & 2,616 & 18,688 & 54,608 \\
\hline 3 & 1,424 & 10,174 & 64,783 \\
\hline 4 & 1,134 & 8,100 & 72,883 \\
\hline 5 & 1,086 & 7,760 & 80,642 \\
\hline 6 & 0,802 & 5,728 & 86,370 \\
\hline 7 & 0,677 & 4,839 & 91,209 \\
\hline 8 & 0,348 & 2,488 & 93,697 \\
\hline 9 & 0,310 & 2,214 & 95,911 \\
\hline 10 & 0,257 & 1,839 & 97,750 \\
\hline 11 & 0,187 & 1,333 & 99,084 \\
\hline 12 & 0,072 & 0,517 & 99,601 \\
\hline 13 & 0,033 & 0,233 & 99,834 \\
\hline 14 & 0,023 & 0,166 & 100,000 \\
\hline \multicolumn{4}{|c}{ Método de extração: análise dos componentes principais } \\
\hline \multicolumn{4}{|c|}{}
\end{tabular}

Fonte: dados da pesquisa (2018)

A análise gerou cinco fatores, utilizando-se o critério do eigenvalue maior que 1 . Os cinco fatores, em conjunto, atualmente explicam $80,64 \%$ da variância total das variáveis analisadas. Analisou-se as cargas fatoriais (nível de relação com o fator) de cada variável em relação aos componentes extraídos, cujas informações estão na tabela 5.

TABLA 5

Matriz de componente rotativa

\begin{tabular}{|c|c|c|c|c|c|}
\hline \multirow{2}{*}{ Variáveis } & \multicolumn{5}{|c|}{ Componente } \\
\hline & 1 & 2 & 3 & 4 & 5 \\
\hline Número de funcionários & 0,961 & $-0,015$ & $-0,053$ & $-0,011$ & $-0,013$ \\
\hline Tamanho da estrutura fisica & 0,815 & 0,342 & 0,227 & 0,179 & 0,064 \\
\hline Investimento em estrutura & 0,045 & 0,123 & 0,870 & 0,137 & 0,030 \\
\hline Número de equipes de saúde da familia & 0,916 & 0,029 & 0,060 & $-0,112$ & $-0,007$ \\
\hline Investimento em insumos e medicamentos & 0,930 & $-0,196$ & 0,014 & $-0,158$ & $-0,052$ \\
\hline Número de atendimentos & 0,968 & 0,116 & 0,008 & 0,041 & 0,073 \\
\hline Resolutividade de pacientes & 0,111 & 0,809 & 0,242 & 0,148 & 0,058 \\
\hline $\begin{array}{l}\text { Médicos com residência em saúde da } \\
\text { familia }\end{array}$ & 0,043 & 0,841 & 0,134 & $-0,257$ & 0,086 \\
\hline Número de dias trabalhados por funcionário & 0,711 & $-0,258$ & 0,209 & 0,295 & $-0,268$ \\
\hline Indice "hiperutilizadores" & 0,332 & 0,303 & $-0,114$ & 0,645 & 0,156 \\
\hline Número de reclamações na ouvidoria & 0,149 & 0,075 & $-0,002$ & $-0,758$ & 0,013 \\
\hline Percentual dos custos fixos & $-0,218$ & 0,790 & 0,027 & 0,205 & $-0,309$ \\
\hline Indice da população atendida & $-0,060$ & $-0,052$ & $-0,015$ & 0,076 & 0,963 \\
\hline Nota no PMAQ & 0,095 & 0,190 & 0,714 & $-0,251$ & $-0,064$ \\
\hline
\end{tabular}

Fonte: dados da pesquisa.

Método de Extração: análise de Componente Principal. Método de Rotação:

Varimax com Normalização de Kaiser. Rotação convergida em 6 iterações.

$\mathrm{Na}$ Tabela 5 observa-se a distribuição das variáveis para os referidos componentes (fatores), que é determinado de acordo com as cargas fatoriais de cada variável. Nenhuma das variáveis apresentou carga fatorial acima de 0,4 para mais de um componente, estando, portanto, dentro dos parâmetros adequados para 
a AF. São relacionadas as variáveis aos fatores a partir da maior carga fatorial apresentada em cada componente. Os valores máximos dessas cargas fatoriais estão destacados em negrito, para facilitar a visualização.

Os resultados da análise fatorial indicaram que as variáveis observadas podem ser explicadas por cinco fatores, dispostos no Tabela 6.

TABLA 6

Representação da composição dos fatores

\begin{tabular}{|c|c|c|c|c|}
\hline $\begin{array}{rr}\text { Fator } 1 \\
\end{array}$ & Fator 2 & Fator 3 & Fator 4 & Fator 5 \\
\hline Número de funcionários & & \multirow{3}{*}{$\begin{array}{l}\text { Investimento } \\
\text { em estrutura }\end{array}$} & \multirow{3}{*}{$\begin{array}{l}\text { Índice } \\
\text { "hiperutilizadores" }\end{array}$} & \multirow{6}{*}{$\begin{array}{l}\text { Índice da } \\
\text { população } \\
\text { atendida }\end{array}$} \\
\hline $\begin{array}{l}\text { Tamanho da estrutura } \\
\text { física }\end{array}$ & $\begin{array}{l}\text { Resolutividade } \\
\text { de pacientes }\end{array}$ & & & \\
\hline $\begin{array}{l}\text { Número de equipes de } \\
\text { saúde da família }\end{array}$ & \multirow{2}{*}{$\begin{array}{l}\text { Médicos com } \\
\text { residência em } \\
\text { saúde da } \\
\text { familia }\end{array}$} & & & \\
\hline $\begin{array}{l}\text { Investimento em } \\
\text { insumos e medicamentos }\end{array}$ & & \multirow{3}{*}{ Nota no PMAQ } & \multirow{3}{*}{$\begin{array}{l}\text { Número de } \\
\text { reclamaçốes na } \\
\text { ouvidoria }\end{array}$} & \\
\hline Número de atendimentos & \multirow{2}{*}{$\begin{array}{l}\text { Percentual dos } \\
\text { Custos fixos }\end{array}$} & & & \\
\hline $\begin{array}{l}\text { Número de dias } \\
\text { trabalhados por } \\
\text { funcionário }\end{array}$ & & & & \\
\hline
\end{tabular}

Fonte: dados da pesquisa (2018)

O Fator 1 agrupa a maior quantidade de variáveis (6), e representa aproximadamente $36,0 \%$ da variância total do modelo. A maior parte das variáveis que compõem o Fator 1 pertencem ao cost driver estrutural "Escala". A única variável que compõe o Fator 1 e não pertence a "Escala" é o "Número de Dias Trabalhados por Funcionário". No contexto desta pesquisa, essa variável foi utilizada para medir o "Comprometimento da Força de Trabalho", entendido como cost driver de execução. Não se pode afirmar que os cost drivers "Escala" e "Comprometimento da Força de Trabalho" estão relacionados. No entanto, pode-se sugerir que, nos centros de saúde, a medida "Número de Dias Trabalhados por Funcionário" esteja mais ligada aos aspectos estruturais de "Escala" do que aos demais. Igualmente, esta variável pode ser um indicativo de dimensão de um centro de saúde em Florianópolis.

O Fator 2 reuniu três variáveis, representando 18,7\% do total da variância do modelo. Duas variáveis representam o cost driver "Experiência": "Resolutividade de Pacientes" e "Médicos com Residência em Saúde da Família”. A terceira variável mede o cost driver "Utilização da Capacidade": "Percentual dos Custos Fixos". No contexto desta pesquisa, os resultados mostram que os aspectos ligados à capacitação da mão de obra estão fortemente associados.

Ainda observando os resultados do Fator 2, deve-se ponderar que as outras variáveis do cost driver "Utilização da Capacidade" foram eliminadas da AF. Desta forma, os resultados da pesquisa sugerem que os cost drivers "Experiência" e "Utilização da Capacidade" estão relacionados no contexto estudado. Uma possível explicação é que os funcionários que têm mais experiência e conhecem melhor os processos dos centros de saúde, também são capazes de melhor utilizar os recursos e instalações disponíveis nessas unidades. No entanto, é preciso ter cautela nessas afirmações, já que as demais variáveis que medem o cost driver "Utilização da Capacidade" foram excluídas da análise. Portanto, não é possível afirmar se, de fato, o "Percentual de Custos Fixos" é um bom indicador para este cost driver.

Em relação ao Fator 3, este representa 10,2\% da variância do modelo e possui duas variáveis: "Investimento em Estrutura" e "Nota no PMAQ". Dos resultados deste fator, destaca-se o fato do "Investimento em Estrutura" não estar relacionado com o Fator 1, em que predominam variáveis do cost driver "Escala”, e sim com o Fator 3. Como os centros de saúde pesquisados são de responsabilidade pública do município, as decisões em torno da construção e/ou ampliação de uma unidade são influenciadas por decisões políticas. Os montantes de investimento nestas unidades podem depender de diferentes políticas orçamentárias para o período no 
qual foram construídas. Neste sentido, não é possível afirmar que um centro de saúde com maior dimensão terá investimento maior, e vice-versa. Apenas se menciona que este indicador está relacionado com outros aspectos, não investigados neste estudo.

O Fator 4 resultou do agrupamento de duas variáveis, ambas do cost driver "Gestão da Qualidade Total", e representa $8,1 \%$ do total da variância do modelo. Os resultados da AF para o fator indicam que as duas variáveis estão fortemente relacionadas entre si, e que, no contexto deste estudo, representam a "Gestão da Qualidade Total" para os centros de saúde de Florianópolis. O Fator 5 evidenciado na AF é composto por apenas uma variável, o "Indice da População Atendida", que representa o cost driver "Eficiência do Layout das Instalações”. Os resultados, porém, apontaram poucas evidências para afirmar que um dos fatores é determinado por este cost driver.

\section{Análise da relação entre os cost drivers e os custos dos centros de saúde}

A partir dos fatores criados pela AF, se estabelece a relação entre os cost drivers e os custos dos centros de saúde. O resumo do modelo é exposto na Tabela 7.

TABLA 7

Resumo do modelo

\begin{tabular}{cccc}
\hline$R$ & $R^{2}$ & $R^{2}$ ajustado & Erro padrão da estimativa \\
\hline $0,982^{\mathrm{e}}$ & 0,965 & 0,958 & $248.561,53$ \\
\hline
\end{tabular}

Fonte: dados da pesquisa.

Pelo resultado de $\mathrm{R}$ se observa que as variáveis do modelo explicam em aproximadamente 95\% o comportamento da variável dependente Custo. Verificando a significância do modelo, o teste ANOVA apresenta resultados que explicam a maior parte da variável dependente. A Tabela 8 mostra os resultados do teste.

TABLA 8

Teste ANOVA

\begin{tabular}{l|r|c|r|r|r}
\hline & $\begin{array}{r}\text { Soma dos } \\
\text { quadrados }\end{array}$ & df & Quadrado médio & F & Sig. \\
\hline Regressão & $4,229 \mathrm{E}+13$ & 5 & $8,458 \mathrm{E}+12$ & 136,9 & 0,00 \\
\hline Resíduo & $1,545 \mathrm{E}+12$ & 35 & $6,178 \mathrm{E}+10$ & - & - \\
\hline Total & $4,384 \mathrm{E}+13$ & 40 & - & - & - \\
\hline
\end{tabular}

Fonte: dados da pesquisa.

Os resultados indicam que o modelo é significativo, pois o valor de Sig. $(0,00)$ é inferior ao nível de significância de 5\%, o que indica que pelo menos uma das variáveis independentes exerce influência sobre o custo. Desta forma, rejeita-se a hipótese nula de que a média de Y (Custo) é melhor para explicar os valores de Y do que o próprio modelo da regressão. Após verificar que o modelo é significativo, demonstra-se os resultados dos coeficientes da regressão, dispostos na tabela 9. 
TABLA 9

Coeficientes da regressão com os fatores

\begin{tabular}{|c|c|c|c|c|c|c|c|}
\hline & \multicolumn{2}{|c|}{ Coeficientes não padronizados } & \multirow{2}{*}{$\begin{array}{r}\begin{array}{r}\text { Coeficientes } \\
\text { padronizados }\end{array} \\
\text { Beta }\end{array}$} & \multirow[t]{2}{*}{$\mathrm{T}$} & \multirow[t]{2}{*}{ Sig. } & \multicolumn{2}{|c|}{ Estatísticas de colinearidade } \\
\hline & B & Erro padrão & & & & Tolerância & VIF \\
\hline Constante & $2.530 .829,89$ & $44.642,97$ & - & 56,69 & 0,000 & - & - \\
\hline Fator 1 & $1.173 .957,66$ & $45.380,92$ & 0,971 & 25,87 & 0,000 & 1,00 & 1,00 \\
\hline Fator 2 & $138.954,01$ & $45.380,92$ & 0,115 & 3,06 & 0,005 & 1,00 & 1,00 \\
\hline Fator 3 & $58.442,95$ & $45.380,92$ & 0,048 & 1,29 & 0,210 & 1,00 & 1,00 \\
\hline Fator 4 & $-48.375,06$ & $45.380,92$ & $-0,040$ & $-1,07$ & 0,297 & 1,00 & 1,00 \\
\hline Fator 5 & $-80.259,07$ & $45.380,92$ & $-0,066$ & $-1,77$ & 0,089 & 1,00 & 1,00 \\
\hline
\end{tabular}

Fonte: dados da pesquisa.

A partir do VIF das variáveis, se observa que não há multicolinearidade no modelo, de acordo com os parâmetros de Gujarati (2000) e Hair, Black, Babin, Anderson e Tathan (2005). Isto se deve ao fato de ter sido realizada a AF previamente, um dos objetivos da qual é eliminar a multicolinearidade.

Em relação aos coeficientes da regressão, o fator que mais influencia os custos nos centros de saúde é o Fator 1 - relacionado predominantemente ao cost driver "Escala". A partir dos resultados pode-se afirmar que, para o contexto dos centros de saúde de Florianópolis, aproximadamente $97 \%$ das variações do Fator 1 explicam as variações no custo destas unidades, com uma confiança maior que 99\%. Esse resultado está alinhado aos achados de Foster e Gupta (1990), Banker e Johnston (1993), Banker et al. (1995) e Horngren et al. (2002), que também verificaram que o cost driver "Escala" - observado pela variável "Volume" - é o que melhor determina o custo das organizações pesquisadas. Isso indica que centros de saúde com maior área de planta, maior número de equipes de saúde e funcionários, que gastam mais insumos e que atendem mais pacientes, de fato apresentam maior custo, sendo que essa relação é explicada em 97\%.

O Fator 2 - representado predominantemente pelas variáveis do cost driver "Experiência" - apresentou um coeficiente padronizado de Beta de 0,12 , o que indica uma relação fraca e positiva, com um nível de significância de 0,5\%, enquanto se esperava o oposto. De acordo com Porter (1985), Pindyck e Rubinfeld (2005) e Souza e Rocha (2009), os custos deveriam diminuir à medida em que uma organização melhora seus processos em razão da maior experiência adquirida. No caso dos centros de saúde de Florianópolis ocorreu o oposto, pois enquanto os números referentes às variáveis de "Experiência" sobem, o custo também sobe, ainda que de forma fraca. No período pesquisado, aproximadamente dois terços dos custos dos centros de saúde foram relativos à remuneração de funcionários, sugerindo que essa relação positiva entre experiência e custo pode ser decorrente do fato de que médicos mais qualificados e com especialização terem remuneração mais alta do que médicos com menor qualificação. Entretanto, isso não se reflete em termos de eficiência nas unidades de saúde, no caso pesquisado. No entanto, em uma instituição de saúde a qualificação dos profissionais pode representar maior custo, mas do ponto de vista do paciente, pode melhorar o atendimento e salvar maior número de vidas.

Os Fatores 3 e 4, representados predominantemente, pelos cost drivers "Configuração do Produto" e “Gestão da Qualidade Total”, apresentaram níveis de significância maiores do que o aceitável. Portanto, sua análise não pôde ser realizada com confiabilidade pois as variações podem ter ocorrido ao acaso, de acordo com os valores de Sig.

O Fator 5 também apresentou valor de Sig. maior do que 0,05, porém, se considerar o modelo com nível de significância de $10 \%$, pode-se inferir que este fator, composto pelo cost driver "Eficiência do Layout das Instalações", possui correlação fraca e negativa com o custo. Portanto, ainda que de forma sutil, esse indicador está alinhado com o que se espera, na visão de Chiavenato (2006), Paranhos (2007) e Martins e Laugeni (2010), onde melhor aproveitamento do layout da organização resulta em redução dos custos. Como os 
valores dos coeficientes foram fracos, bem como do nível de significância para este fator, tais constatações precisam de análise mais cautelosa.

Desta forma, os resultados da regressão para os dados dos centros de saúde e período pesquisados sugerem que: (i) o cost driver estrutural "Escala" impacta positivamente e de maneira forte os custos dos centros de saúde; (ii) o cost driver estrutural "Experiência" impacta positivamente e de maneira fraca os custos dos centros de saúde; e (iii) o cost driver de execução "Eficiência do Layout das Instalações" impacta negativamente e de maneira fraca os custos dos centros de saúde.

Os demais cost drivers que foram abordados nesta pesquisa não obtiveram evidências suficientes para explicar os custos dos centros de saúde pesquisados, com as ferramentas estatísticas empregadas. A fim de sintetizar os resultados encontrados e fornecer um panorama geral em relação aos cost drivers estratégicos e seu impacto no custo dos centros de saúde de Florianópolis, a Tabela 10 apresenta o resumo dos resultados das análises.

TABLA 10

Síntese dos resultados da pesquisa

\begin{tabular}{|c|c|c|c|}
\hline \multirow{2}{*}{ Fator } & \multirow{2}{*}{ Cost driver relacionado } & \multicolumn{2}{|c|}{ Influência no custo } \\
\hline & & Direção & Intensidade \\
\hline & $\begin{array}{l}\text { Escala } \\
\end{array}$ & & \\
\hline Fator 1 & $\begin{array}{l}\text { Envolvimento da força de } \\
\text { trabalho }\end{array}$ & Positiva & Forte \\
\hline Fator 2 & $\begin{array}{l}\text { Experiência } \\
\text { Utilização da capacidade }\end{array}$ & Positiva & Fraca \\
\hline Fator 3 & $\begin{array}{l}\text { Escala } \\
\text { Configuração do produto }\end{array}$ & \multicolumn{2}{|c|}{ Não significativa } \\
\hline Fator 4 & Gestão da qualidade total & \multicolumn{2}{|c|}{ Não significativa } \\
\hline Fator 5 & $\begin{array}{l}\text { Eficiência do layout das } \\
\text { instalaçốes }\end{array}$ & Negativa & Fraca \\
\hline
\end{tabular}

Fonte: dados da pesquisa.

A relação dos cost drivers com o custo foi estabelecida a partir do agrupamento em fatores. Os resultados mostram que do total de cinco fatores, dois não obtiveram significância estatística suficiente para estabelecer sua relação com o custo. Dos demais, o Fator 1 foi o que mais impactou nos custos dos centros de saúde de Florianópolis, com sua relação positiva e forte. Desta forma, o cost driver "Escala" se mostrou o mais influente, assim como nos resultados dos estudos de Foster e Gupta (1990), Banker e Johnston (1993), Banker et al. (1995) e Horngren et al. (2002).

Os resultados permitem inferir que o custo é mais sensível, no contexto desta pesquisa, a aspectos estruturais como o tamanho do centro de saúde ou o número de funcionários, e que envolvem decisões tomadas a partir da demanda de cada região. Portanto, se uma unidade possui apenas uma equipe de saúde da família e precisa, por exemplo, aumentar para duas equipes, seu impacto no custo será sentido rapidamente. A remuneração aumentará já no mês seguinte, pois remuneração representa o maior custo das unidades.

Em contrapartida, o impacto da "Experiência" e da "Eficiência do Layout das Instalações" (Fatores 2 e 5 ) nos custos é sentido de maneira menos imediata. Os resultados indicam que esses cost drivers afetam de maneira mais lenta e em menor proporção os custos, pois envolvem aspectos que ocorrem de maneira gradual e no longo prazo. 


\section{CONCLUSÕES DO TRABALHO}

Este estudo teve o objetivo de identificar os fatores que influenciam os custos, na abordagem da GEC, nos centros de saúde administrados pela Secretaria Municipal de Saúde de Florianópolis, SC, Brasil. O Análise Fatorial indicou que as variáveis identificadas para os centros de saúde podem ser explicadas por cinco fatores, sendo mais representativo o Fator 1, relacionado predominantemente ao cost driver estrutural "Escala". O Fator 1 também incluiu o maior número de variáveis na pesquisa, devido à maior facilidade de medição e disponibilidade de dados na SMS. Igualmente, esse fator integrou uma variável do cost driver de execução "Comprometimento da Força de Trabalho", mas verificou-se maior relação desta com aspectos de "Escala" dos centros de saúde. Duas linhas de explicação se sugerem para este último fenômeno: i) a variável utilizada para medir o "Comprometimento da Força de Trabalho" não está adequada, ou seriam necessárias mais variáveis para que essa análise tivesse mais subsídio. Pode se considerar esta falta de adequação como uma limitação deste estudo; ii) pode se sugerir que, de fato, as variáveis "Escala" e "Comprometimento da Força de Trabalho" estão relacionadas.

Quanto à relação entre os cost drivers e o custo dos centros de saúde pesquisados, os resultados sugerem que: (i) o cost driver estrutural "Escala" impacta positivamente e de maneira forte os custos dos centros de saúde; (ii) o cost driver estrutural "Experiência" impacta positivamente e de maneira fraca os custos; e (iii) o cost driver de execução "Eficiência do Layout das Instalações" impacta negativamente e de maneira fraca os custos dos centros de saúde.

Os resultados sugerem, portanto, que o custo é mais sensível e responde mais rapidamente a mudanças nas variáveis estruturais que envolvem "Escala". Por sua natureza, este cost driver está diretamente relacionado à prestação de serviços de saúde. Assim, os centros de saúde que possuem maior demanda de pacientes irão gerar maiores custos, pois eles necessitam de estruturas maiores, mais equipes de saúde da família e o provimento de capacidade para atender maior demanda. Em contrapartida, "Experiência" e "Eficiência do Layout das Instalações" atuam sobre uma parcela menor do custo e têm impacto percebido de forma menos intensa. Isto se deve a que as mudanças ocorridas pela melhoria dos processos em função do aprendizado e os ajustes em relação ao layout das instalações têm impacto menor sobre o total dos custos, configurando um menor impacto marginal.

Os resultados deste estudo foram gerados pela análise de vinte variáveis, distribuídas em nove cost drivers. No entanto, alguns não puderam ser observados, como é o caso da "Complexidade" e da "Exploração das Ligações da Cadeia de Valor”. Outros, entretanto, foram representados por poucas variáveis, devido à característica da captação de dados dos centros de saúde de Florianópolis, o que também caracteriza uma limitação deste estudo.

Os resultados desta pesquisa foram obtidos no contexto específico dos centros de saúde municipais, diferente de estudos prévios, realizados em indústrias. Outros cost drivers podem afetar o custo dessas instituições, mas não puderam ser identificados nesta pesquisa por limitações da base de dados ou pelos métodos estatísticos utilizados. Tal é o caso de "Tecnologia" e "Complexidade", presentes em outros estudos como em Banker e Johnston (1993), Datar et al. (1993), Alcouffe et al. (2010) e Diehl et al. (2010).

Sugere-se, para estudos posteriores, que sejam melhoradas as formas de mensuração das variáveis que definem os cost drivers, para que possam ser observados com maior detalhamento. Igualmente, se recomenta integrar cost drivers não abordados nesta pesquisa e, assim, apresentar mais evidências a respeito de sua influência nos custos das organizações. Outra recomendação é que seja realizado um estudo de caso em um ou mais centros de saúde com o intuito de melhorar as formas de mensuração para as variáveis de avaliação dos cost drivers, e possibilitar deste modo que as relações encontradas nesta pesquisa sejam analisadas com maior profundidade. 


\section{REFERÊNCIAS}

Alcouffe, S., Berland, N., Dreveton, B. \& Essid, M. (2010). An empirical study of environmental cost drivers. Crises et nouvelles problématiques de la Valeur.

Alemi, F. \& Sullivan, T. (2007). An example of activity-based costing of treatment programs. American Journal of Drug and Alcohol Abuse, 33, 89-99. https://doi.org/10.1080/00952990601087372

Anderson, S. (2007). Managing costs and cost structure throughout the value chain: Research on strategic cost management. Handbook of Management Accounting Research, 2, 481-506. https://doi.org/10.1016/S1751-324 3(06)02001-3

Banker, R. \& Johnston, H. (1993). An empirical study of cost drivers in the US airline industry. Accounting Review, 68(3), 576-601.

Banker, R. \& Johnston, H. (2007). Cost and profit driver research. Handbooks of Management Accounting Research, 2, 531-556. https://doi.org/10.1016/S1751-3243(06)02003-7

Banker, R., Potter, G. \& Schroeder, R. (1995). An empirical analysis of manufacturing overhead cost drivers. Journal of Accounting and Economics, 19. https://doi.org/10.1016/0165-4101(94)00372-C

Bezerra, F. (2014). Análise Fatorial. En L. Corrar et al. Análise multivariada para cursos de administração, ciências contábeis e economia. São Paulo: Atlas.

Blocher, E., Chen, K. \& Lin, T. (2008). Cost management: A strategic emphasis. Irwin: McGrawHill.

Bonacim, C. \& Araújo, A. (2010). Gestão de custos aplicada a hospitais universitários públicos: a experiência do Hospital das Clínicas da Faculdade de Medicina de Ribeirão Preto da USP. Revista de Administração Pública, 44(4), 903-931.

Cardinaels, E., Roodhooft, F. \& Herck, G. (2004). Drivers of cost system development in hospitals: results of a survey. Health Policy, 69, 239-252. https://doi.org/10.1016/j.healthpol.2004.04.009

Chiavenato, I. (2006). Administração Geral e Pública. Rio de Janeiro: Elsevier.

Cooper, R. \& Kaplan, R. S. (1987). How cost accounting systematically distorts product costs. Accounting and management: Field study perspectives, p. 204-228.

Cooper R. \& Kaplan R. S. (1998). The Design of Cost Management Systems: Text, Cases and Readings. 2. Ed.

Costa, S. \& Rocha, W. (2014). Determinantes de custos de concorrentes: identificação a partir de informações públicas. Revista de Gestão e Contabilidade da UFPI, 1(1), 4-24.

Diehl, C., Miotto, G. \& Souza, M. (2010). Análise da tecnologia das aeronaves como determinante de custos no setor de aviação comercial brasileiro. Revista Brasileira de Gestão de Negócios - FECAP, 12(35), Abr./Jun.

Datar, S., Kekre, S., Mukhopadhyay, T. \& Srinivasan, K. (1993). Simultaneous Estimation of Cost Drivers. The Accounting Review, 68(3), 602-614.

Dieng, M., Araujo, A., Diniz, J., Diniz, M. \& Santos, D. (2006). Gestão Estratégica de Custos Aplicada à Atividade Hoteleira: um estudo Empírico nos Hotéis de Médio e Grande Porte da Grande Recife. Congresso USP de Controladoria e Contabilidade. Anais... São Paulo: Universidade de São Paulo.

Foster, G. \& Gupta, M. (1990). Manufacturing overhead cost driver analysis. Journal of Accounting and Economics, 12(1-3), 309-337. https://doi.org/10.1016/0165-4101(90)90052-6

Gil, A. (2002). Como elaborar projetos de pesquisa, 4. ed., São Paulo: Atlas.

Gujarati, D. (2000). Econometria básica, 3 ed. São Paulo: Makron Books.

Hair Jr., J., Black, W., Babin, B., Anderson, R. \& Tathan, R. (2005). Análise multivariada de dados, 5. ed. Porto Alegre: Bookman.

Hansen, D. \& Mowen, M. (2005). Cost Management: Accounting and Control. Cengage Learning.

Henri, J., Boiral, O. \& Roy, M. (2015). Strategic cost management and performance: The case of environmental costs. British Accounting Review, 48(2), 269-282. https://doi.org/10.1016/j.bar.2015.01.001 
Horngren, C., Bhimani, A., Datar, S. \& Foster, G. (2002). Management and cost accounting. Harlow: Financial Times / Prentice Hall.

Horngren, C., Datar, S. \& Foster, G. (1999). Contabilidade de Custos, 9 ed. Rio de Janeiro: Editora LTC SA.

Hsu, S. \& Qu, S. (2012). Strategic cost management and institutional changes in hospitals, European Accounting Review, 21(3), 499-453. https://doi.org/10.1080/09638180.2012.658152

IFAC - International Federation of Accountants. (1998). International management accounting practice 1 (IMAP1).

Instituto Brasileiro de Geografia e Estatística. (2017). Conta-Satélite de Saúde 2010-2015: em 2015, 9,1\% do PIB foram gastos no consumo de bens e serviços de saúde. Estatísticas Econômicas. Disponível em: https://agenciadenoticias.ibge.gov.br/agencia-noticias/2013-agencia-de-noticias/releases/18915-conta-sat elite-de-saude-release.html. Acesso em: fevereiro/2018.

Johnson, H. (1992) Relevance regained: from top-down control to bottom-up empowerment. New York: Free Press.

Johnson, H. \& Kaplan, R. (1987). Relevance lost: The rise and fall of management accounting. Boston: HBSP.

Kaplan, R. (1983). Measuring manufacturing performance: a new challenge for managerial accounting research. The Accounting Review, 58, 686-705.

Kaplan, R. (1984). Yesterday's accounting undermines production. Harvard Business Review, 84(4), 95-101.

Liu, Q. (2015). Research on Strategic Cost Management in Modern Enterprises. Management \& Engineering, $21,32$. DOI: https://doi.org/10.5503/J.ME.2015.21.005

Martins, P. \& Laugeni, F. (2010). Administração da produção, 2. ed. São Paulo: Saraiva.

Miller, J. \& Vollmann, T. (1985). The hidden factory. Harvard Business Review. Sep./Oct.

Ministério da Saúde. (2008). Manual de estrutura física das unidades básicas de saúde. Brasília.

Morse, W., Davis, J. \& Hartgraves, A. (2002). Management Accounting: A Strategic Approach. South-Western College.

Niu, L., Chen, M., Jiang, J. \& Zhou, Q. (2012). The drivers analysis of strategic cost based on AHP. In: Advances in Electrical Engineering and Automation (pp. 59-65). Springer Berlin: Heidelberg.

Paranhos Filho, M. (2007). Gestão da Produção Industrial. Curitiba: Ibpex.

Pindyck, R. \& Rubinfeld, D. (2005). Microeconomia, 6. ed. Tradução de Eleutério Prado e Thelma Guimarães. São Paulo: Prentice Hall.

Porter, M. (1985). Competitive advantage: creating and sustaining superior performance. New York: FreePress.

Richardson, R. (2008). Pesquisa social: métodos e técnicas, 3. ed. São Paulo: Atlas.

Riley, D. (1987). Competitive cost based investment strategies for industrial companies. In Manufacturing Issues, New York: Booz Allen and Hamilton.

Shank, J. (1989). Strategic Cost Management: New wine, or just new bottles? Journal of Management Accounting Research. Vol. 1.

Shank, J. \& Govindarajan, V. (1989). Strategic cost analysis: The evolution from managerial to strategic accounting. Homewood, IL: Irwin.

Shank, J. \& Govindarajan, V. (1997). A revolução dos custos: Como reinventar e redefinir sua estratégia de custos para vencer em mercados crescentemente competitivos. Editora Campus: São Paulo.

Schultz, C., Marques, T., Borgert, A. \& Rocha, R. (2012). Publicações sobre acitivity-based costing na área de cuidados em saúde. Em XXXII Encontro Nacional de Engenharia de Producao, , Anais... Bento Gonçalves.

Slavov, T. \& Takahashi, M. (2010). Além do direcionamento de custos: a visão dos determinantes de custos - cost drivers - na gestão estratégica de custos. Congresso Brasileiro de Custos. Anais... Belo Horizonte.

Souza, B. \& Rocha, W. (2009). Gestão de Custos Interorganizacionais. São Paulo: Atlas.

Vergara, S. (1997). Projetos e relatórios de pesquisa em administração. São Paulo: Atlas. 


\section{Notas}

* Pesquisa científica e tecnológica.

\section{Licencia Creative Commons CC BY 4.0}

Para citar este artigo: Borgert, E. A., \& Gasparetto, V. (2019). Fatores que influenciam os custos, na abordagem da gestão estratégica de custos: análise empírica em centros de saúde municipais. Cuadernos de Contabilidad, 20(49). https://doi.org/10.11144/Javeriana.cc20-49.fica 